Kalpa Publications in Engineering
Volume 2, 2018, Pages 7-14
Proceedings on International Conference on Emerg-
ing Trends in Expert Applications \& Security (2018)

\title{
A Review of Face Recognition System Using Raspberry Pi in the Field of IoT \\ Arihant Kumar Jain ${ }^{1}$, Richa Sharma ${ }^{2}$, Anima Sharma ${ }^{3}$ \\ 1,2,3 Jaipur Engineering College \& Research Centre, Jaipur \\ arihantjain.csedjecrc.ac.in, \\ richasharmacse@jecrc.ac.in, animasharma.csedjecrc.ac.in
}

\begin{abstract}
In these days circumstance, the security frames the most essential segment of our lives. Security of the house or the close what's more, dear ones is critical to everyone. Home computerization is an energizing zone for security applications. This field has improved with new advances such Internet of things (IoT). In IoT, each device carries on as a little piece of a web hub and each hub associate and convey. Of late, surveillance cameras are used keeping in mind the end goal to construct security spots, homes, and urban communities. Be that as it may, this innovation needs a man who recognizes any issue in the edge taken from the camera. In this paper, an Internet of Things is joined with PC vision so as to identify the characteristics of individuals. For this reason, to execute this framework, a charge card measure PC that uses its own particular camera board for the security framework, for example, raspberry pi 3 used. In like manner, Passive Infrared Sensor (PIR) mounted on the Raspberry PI is used to identify any developments. So it screens and gets warnings when movement is distinguished, catches the picture and identify the faces, at that point sends pictures to a Smartphone by means of using wire application. Internet of things in light of wire application used to see the action and get sees when development is distinguished.
\end{abstract}

Index Terms- Face Identification, Raspberry PI, Computer Vision, Internet of things, wire.

\section{Introduction}

Nowadays, humans live in the information period. There are various ways in daily life for preparing to the Internet and to make our day by day life simpler and more pleasing like Smartphone, computers, tablets, smart cars and some Smart TVs. The new gadgets can run any projects, in much best and more viable path for doing diverse errands for example power on or power off the gadget, making cautions through the builtin or outer sensors. These items can be characterized as Smart Objects. Smart Objects can be more helpful to each other and with other objects, for example actuators or sensors. In like manner, smart systems can be given IoTs and besides allowed creating either large or small system due to obtain an aggregate intelligence through the processing of object's information [1]. The IoTs can be connected in Smart Cities in order to give variety advantages that improve resi- 
dents [2- 3]. In different terms, brilliant homes can be made through using the Internet of Things which can control and robotize correct things of our houses for example doors, lights, windows, distributed multimedia, fridges, and irrigation systems. Computer Vision gives face detection and recognition for people that are an extremely fascinating application for the Internet of Things. Computer Vision combinations can exhibit greater security system in an IoT platform for intelligent homes since they have capacities to perceive a human in the incorrect place since this human might be a bad human for the environment [4].

There will be combination of wide-ranging smartly linked devices that will further expand the boundaries of universe, having variety of environment/context, objects and frameworks in the new generation of Internet. The Internet of Things (IoT) is basically a phrase that describes a network of physical and virtual devices/equipments encapsulated with electronics, software, sensors, actuators linked with network to be able to communicate and identified by each other. The concept of IoT is vital because when an object is no longer/(heretofore) connected to its user can still be connected to ambient objects and database data. In today's era security \& piracy are the major issues so lot of research is going on. Facial recognition using IoT is one of the areas where lot of work has been done. In this system facial image is detected and identified \& user is informed accordingly through a text message, email or some audio message. Advance knowledge of microcontrollers and interfaces such as Raspberry Pi is used to design such type of system.

Monitoring assumes an exceptionally important role to fulfill our safety. Security system sensors assume a very important role in this area. There are two principle kinds of sensors: indoor sensors which ensure and protect the inside of the home and sensors that ensure and protect the outdoor of the home. The most widely sensors are microwave sensor, Passive Infrared Sensor (PIR), photo-electric sensor, noise detector, and ultrasonic sensor. The wireless system gives a capable, stylish and strong solution for the problem of remote house access, safety, and monitoring with face detection. Real time face detection systems are important for photography, security, and surveillance applications [5]. Mostly, it perceives and track people in public place like homes, airports, shopping centers, offices, bank, in regions with limited access sensors. There are some documents proposed for the field of security system. Every security system applications are used for different purpose, and in the writing, there are a small amount of studies through utilizing a camera in IoTs and sensors. The Internet of Things could help to learn and demonstrate different information amongst master and understudies through gathering information and find the best route to train. Thus, they can assistance to protect the right and people of cities. Creating or studying on maps, which is called Cartography, is another utilization of Computer Vision. The most famous sample of cartography is Bing Maps or Google Maps, which is a useful service for people. Along these lines, Computer Vision is programmed for perceiving some specific parts in maps: for example roads, water, buildings, or fields. This is an instance of how to relate Computer Vision in Smart Earth [2]. Prof. Vijay Bagdihave, Ms. RenukaChuimurkar showed a smart monitoring system utilizing PIR sensor, Raspberry Pi, and mobile device. They likewise should utilize smoke detector for detecting fire. After capturing the image to customer mail via Wi-Fi, user will be alerting about the fire or thief. They used smoke discovery algorithm and background subtraction algorithm for motion detection [6]. The image of a person sent to twitter and email after captured [7].

In this paper, Raspberry PI 3 is used and PICAMERA associated to it, the application will take a picture immediately after PIR sensor finds any motion. After, we apply haar cascade algorithm to detect the faces in the captured image. Then, we send these pictures to a Smart phone through the internet. For this situation, Internet of things established telegram application used to see the action and get notifications and pictures. The Adaboost learning is used to detect the face from theHaar-like features 
called as the Viola-Jones technique. In general, the face detection method is established on the ViolaJones method. It gives better detection rate by comparison to other methods. The calculation cost of the Viola-Jones method is relative low. Along these lines, this face detection strategy becomes the most superb technique. However, when the face is overlapping by the other objects the technique fails to detect the face [8].

\section{The Internet of Things}

These days, the Internet of Things is rapidly increasing technology with business situation. IoT is the conjunction of internet, wireless network, and computing. As of late, the Internet of Things is one of the mainly used technology together benefit for a little country. IoT connects the physical things like vehicles, buildings, and various devices with embedded intelligent sensors and empowers these objects to replace and collect data [9-12]. The IoT is becoming popular in many sides of life, such as smart security, smart cities, health care, smart transportation, smart grids, online business, and so on. Our target in using Internet of Things is to share our data and learning to everybody in wherever around the earth, from food to computer's and computerize diverse actions to improve our day by day living $[2,13]$. The IoT is an active overall system of day by day objects linking to the internet. The IoT is the interconnection of physical and virtual things oncoming via the internet. The IoT is the name of internet of objects, belongs to a wireless network among objects. The Internet of Things let making a Smart World, which is the fusion of different and ubiquitous things, Smart Homes, and Smart Cities, with all gadgets fit for cooperating amongst themselves [2]. Wireless Sensor Networks is the most essential, which are the center of the Internet of Things. A Wireless Sensor Network (WSN) interconnects sensors, in order to obtain data, by a server or unique scheme to work and may be, computerize everyday jobs in one position [14]. The best definition for IoT is that it is an interconnection of different and ubiquitous objects among themselves via the Internet. The aim of the IoT is to be linked the whole world through the building of diverse intelligent areas to computerize, facilitate and improve our routine life [15]. These days, IoT is turn into a part of all part of our lifetime. IoT applications not only enhance the convenience of our lifetime. Besides, it allows us too much control through simplify style work days and task of personals. The application areas of IoT are given in Fig. 1.

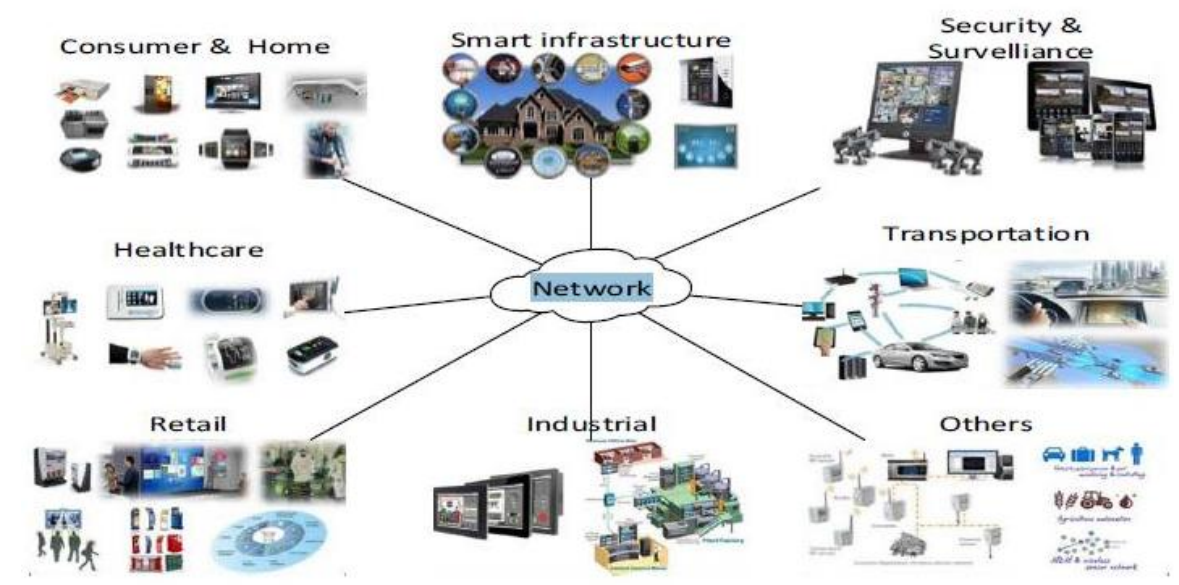

Fig.1. The application areas of IoT 
In Fig.1, IoT has been used in many application areas. Some of these IoT platforms have attributes that others don't have. Be that as it may, none of them has a module of Computer Vision that permits working with images as sensors and images with sensors. Our proposal is one possible solution to utilize the camera with a sensor utilizing Computer Vision to detect a specified thing in images.

\section{Face Recognition}

Face recognition has been an active research topic since the 1970's. Given an input image with multiple faces, face recognition systems typically first run face detection to isolate the faces. Each face is preprocessed and then a low-dimensional representation (or embedding) is obtained. A lowdimensional representation is important for efficient classification. Challenges in face recognition arise because the face is not a rigid object and images can be taken from many different viewpoints of the face. Face representations need to be resilient to intrapersonal image variations such as age, expressions, and styling while distinguishing between interpersonal image variations between different people. To summarize the regimes, face recognition research can be characterized into feature based and holistic approaches.

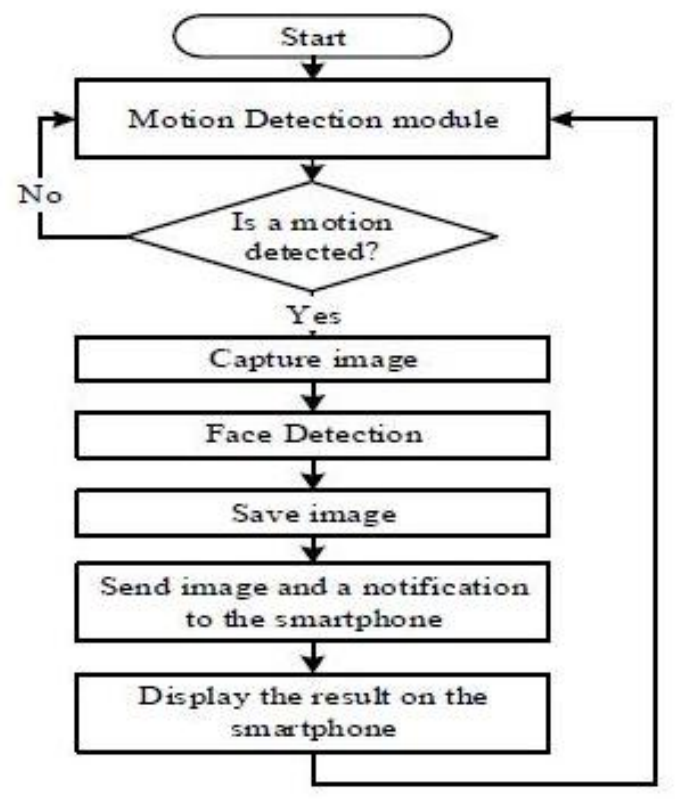

Fig.2. Flowchart for proposed system

\section{Review of methods for face detection}

In the proposed system, we use the camera to accomplish the pictures when a motion detect via PIR sensor. Subsequently, we will apply computer vision module to the caught pictures to discover the faces, after that, we will mail it to Smartphone. This system is extremely helpful and vital if we want to protect an area. The application can be divided into two parts which are motion detection and face detection. The system will not go to face detection if there is no motion discovered. But, if a movement has detected, then the detected movement of the current frame will be processed by the al- 
gorithm of face detection. Fig. 2 demonstrates the flow chart for the proposed scheme. To explicitly define a low-dimensional face representation based on ratios of distances, areas, and angles An explicitly defined face representation is desirable for an intuitive feature space and technique. However, in practice, explicitly defined representations are not accurate. Later work sought to use holistic approaches stemming from statistics and Artificial Intelligence (AI) that learn from and perform well on a dataset of face images. Statistical techniques such as Principal Component Analysis represent faces as a combination of eigenvectors. However, these deep neural network-based techniques are trained with private datasets containing millions of social media images that are orders of magnitude larger than available datasets for research.

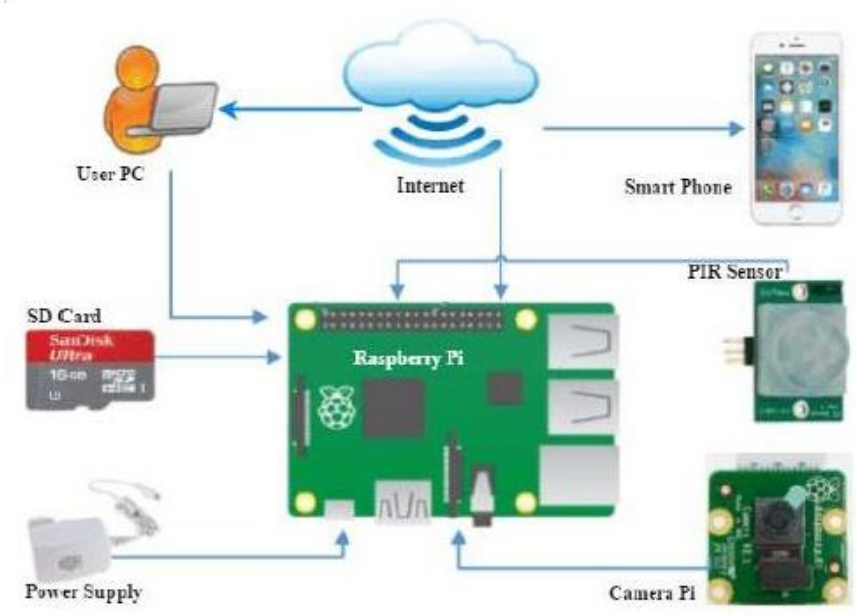

Fig.3. System design

\section{Face recognition for neural network}

This section provides a deeper introduction to face recognition with neural networks from the techniques used in Facebook's DeepFace and Google's FaceNet systems that are used within OpenFace. This section is intended to give an overview of the two most impactful works in this space and is not meant to be a comprehensive overview of the thriving field of neural network-based face recognition. Other notable efforts in face recognition with deep neural networks include the Visual Geometry Group (VGG) Face Descriptor and Lightened Convolutional Neural Networks (CNNs) which have also released code. 


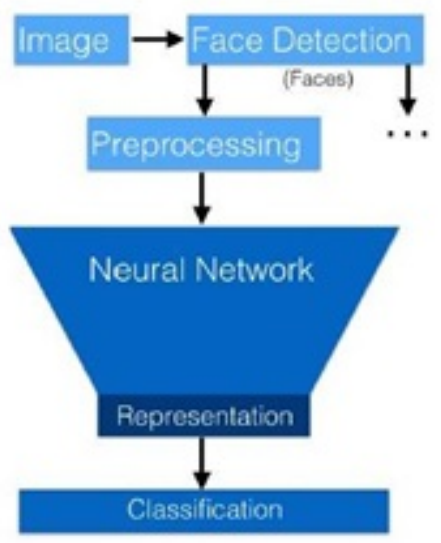

Figure 4 Logic flow for face recognition with a neural network.

A feed-forward neural network consists of many function compositions, or layers, followed by a loss function $\mathrm{L}$ as shown in Figure 1. The loss function measures how well the neural network models the data, for example how accurately the neural network classifies an image. Each layer I is parameterized by i, which can be a vector or matrix. Common layer operations are: Spatial convolutions that slide a kernel over the input feature maps, Linear or fully connected layers that take a weighted sum of all the input units, and Poolingthat take the max, average, or Euclidean norm over spatial regions.

Figure 2 shows the logic flow for face recognition with neural networks. There are many face detection methods to choose from, as it is another active research topic in computer vision. Once a face is detected, the systems preprocess each face in the image to create a normalized and fixed-size input to the neural network. The preprocessed images are too high-dimensional for a classifier to take directly on input. The neural network is used as a feature extractor to produce a low dimensional representation that characterizes a person's face. A low-dimensional representation is key so it can be efficiently used in classifiers or clustering techniques. 


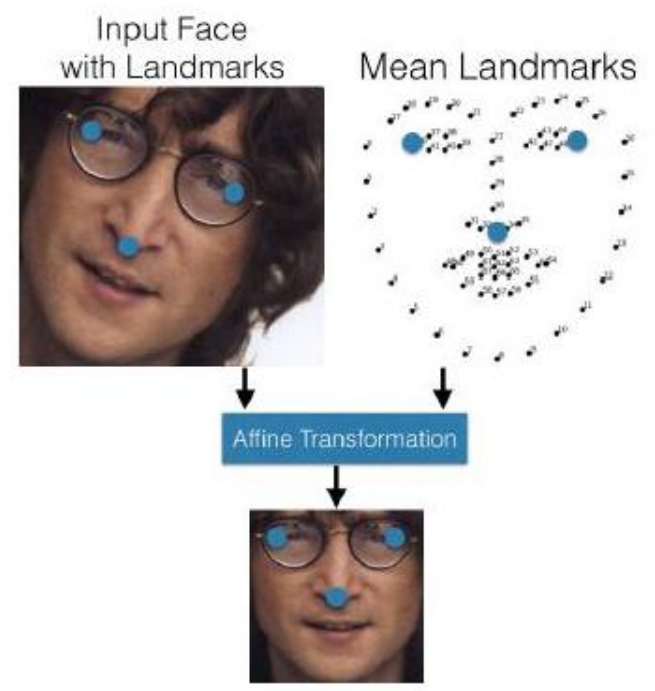

Figure 5: OpenFace's affine transformation. The transformation is based on the large blue landmarks and the final image is cropped to the boundaries and resized to $96 \times 96$ pixels.

\section{Conclusion}

Internet of things in light of wire application used to see the action and get sees when development is distinguished. Computer Vision gives face detection and recognition for people that are an extremely fascinating application for the Internet of Things. Computer Vision combinations can exhibit greater security system in an IoT platform for intelligent homes since they have capacities to perceive a human in the incorrect place since this human might be a bad human for the environment.

\section{References}

[1] C. G. García, D. M. Llorian, B.C.P. G-Bustelo, J.M.C. Lovelle, "A review about smart objects, sensors, and actuators,” Int. J. Interact. Multimed.Artif.Intell., vol. 4, pp. 7-10, 2017.

[2] A.J. Jara, Y. Sun, H. Song, R. Bie, D. Genooud, Y. Bocchi, "Internet of things for cultural heritage of smart cities and smart regions," in: 2015 IEEE 29th Int. Conf. Adv. Inf. Netw. Appl. Work, IEEE, Gwangiu, 2015, pp. 668-675.

[3] H. Gu, D. Wang, “A content-aware fridge based on RFID in smart home for homehealthcare," in: Adv. Commun. Technol. 2009. ICACT 2009. 11th Int. Conf., Phoenix Park, 2009, pp. 987-990.

[4] P.N. Belhumeur, J.P. Hespanha, and D. Kriegman, "Eigenfaces vs. fisherfaces: recognition using class spe- cific linear projection," IEEE Transactions on Pattern Analysis and Machine Intelligence, vol. 19, no. 7, pp. 711-720, 2007. 
[5] C G. S. Arias, C. G. García, B. Cristina, P. G. Bustelo, "Midgar: Study of communications security among Smart Objects using a platform of heterogeneous devices for the Internet of Things" Future Generation Computer Systems, Vol. 74, pp. 444-466, September 2017.

[6] Ms. RenukaChuimurkar, V. Bagdi, "Smart Surveillance Security \&Monitoring System Using Raspberry PI and PIR Sensor", International Journal of Scientific Engineering and Applied Science (IJSEAS), Vol. 2, No. 1, pp. 1-4, January 2016.

[7] Md. NasimuzzamanChowdhury, Md. ShibleeNooman, SrijonSarke, "Access Control of Door and Home Security by Raspberry Pi Through Internet", International Journal of Scientific \& Engineering Research, Vol. 4, pp. 550-558, November-2013.

[8] A. Das, M. Pukhrambam, A. Saha, "Real-time robust face detection and tracking using extended haar functions and improved boosting algorithm", in: 2015 International Conference on Green Computing and Internet of Things (ICGCIoT), pp. 981- 985, 2015.

[9] J.A. Stankovic, "Research directions for the Internet of things", IEEE Trans. On Internet of Things, vol. 1, pp. 3-9, 2014. [10] T. Polk, S. Turner, "Security challenges for the Internet of things," in: Work. Interconnecting Smart Objects with Internet, Prague, 2011.

[11] K.T. Nguyen, M. Laurent, N. Oualha, "Survey on secure communication protocols for the Internet of things," Ad Hoc Networks, Vol. 32, pp. 17-31, September 2015.

[12] International Telecommunication Union, Overview of the Internet of things, 2012, 14. https://www.itu.int/rec/T-RECY. 2060-201206-I.

[13] Vienna University of Technology, European Smart Cities, 2015.http://www.smart-cities.eu (accessed 28.09.16).

[14] T. Polk, S. Turner, "Security challenges for the Internet of things," in: Work. Interconnecting Smart Objects with Internet, Prague, 2011.

[15] P. Martinez-Julia, E.T. Garcia, J.O. Murillo, A.F. Skarmeta, "Evaluating video streaming in network architectures for the Internet of things," in: 2013 Seventh Int. Conf. Innov. Mob. Internet Serv. Ubiquitous Comput., IEEE, Taiwan, 2013, pp. 411-415.

[16] Y. W. Bai, L. SihShen, Z.-H. Li, "Design and Implementation of an Embedded Home Surveillance System by Use of Multiple Ultrasonic Sensors," IEEE Transactions on Consumer Electronics, vol. 56, pp. 119 - 124, March 2010. 